\title{
Two-loop virtual light-by-light scattering corrections to the bound-electron $g$ factor
}

\author{
V. Debierre $\odot,{ }^{1, *}$ B. Sikora, ${ }^{1}$ H. Cakir $\odot,{ }^{1}$ N. S. Oreshkina $\odot,{ }^{1}$ V. A. Yerokhin $\odot,{ }^{1,2}$ C. H. Keitel $\odot,{ }^{1}$ and Z. $\operatorname{Harman}{ }^{1}$ \\ ${ }^{1}$ Max Planck Institute for Nuclear Physics, Saupfercheckweg 1, D-69117 Heidelberg, Germany \\ ${ }^{2}$ Center for Advanced Studies, Peter the Great St. Petersburg Polytechnic University, 195251 St. Petersburg, Russia
}

(Received 23 July 2020; revised 23 November 2020; accepted 17 February 2021; published 10 March 2021)

\begin{abstract}
A critical set of two-loop quantum electrodynamics corrections to the $g$ factor of hydrogenlike ions is calculated in the Furry picture. These corrections are due to the polarization of the external magnetic field by the quantum vacuum, which is dressed by the binding field. The result obtained for the self-energy-magnetic-loop diagrams is compared with the current state-of-the-art result, derived through a perturbative expansion in the binding strength parameter $Z \alpha$, with $Z$ the atomic number and $\alpha$ the fine-structure constant. Agreement is found in the $Z \rightarrow 0$ limit. However, even for very light ions, the perturbative result fails to approximate the magnitude of the corresponding correction to the $g$ factor. The total correction to the $g$ factor coming from all diagrams considered in this work is found to be highly relevant for upcoming experimental tests of fundamental physics with highly charged ions.
\end{abstract}

DOI: 10.1103/PhysRevA.103.L030802

Introduction. Measurements of the $g$ factor of heavy hydrogenlike ions are projected at different facilities, such as the ALPHATRAP Penning trap [1-3] and the HITRAP facility [4-6]. These measurements are forecast to match the most precise measurements of the $g$ factor so far $[7,8]$, which have an uncertainty of the order of $10^{-11}$. High-precision calculations and measurements of the bound-electron $g$ factor can be combined to perform state-of-the-art determinations of fundamental constants such as the electron mass $m_{e}$ [8] and the fine-structure constant $\alpha$ [9-11]. Furthermore, heavy ions are an ideal testing ground for quantum electrodynamics (QED) calculations in the presence of strong fields [7,12-15], and measurements of their $g$ factor were recently shown to be a promising avenue in the search for physics beyond the Standard Model [16].

The interpretation of upcoming experiments on heavy hydrogenlike ions demands improvements in the theory, especially concerning the calculation of radiative corrections to the $g$ factor. The one-loop radiative corrections have been calculated nonperturbatively [17-21] in the electromagnetic binding parameter $Z \alpha$, but the calculation of the two-loop corrections has only been completed through orders $(Z \alpha)^{4}$ $[22,23]$ and $(Z \alpha)^{5}[23,24]$. Further progress of the two-loop calculation should be sought in the nonperturbative approach, especially for application to heavy ions. The nonperturbative evaluation of all 29 nonequivalent two-loop diagrams contributing to the $g$ factor of a bound electron is one of the great

\footnotetext{
*vincent.debierre@mpi-hd.mpg.de
}

Published by the American Physical Society under the terms of the Creative Commons Attribution 4.0 International license. Further distribution of this work must maintain attribution to the author(s) and the published article's title, journal citation, and DOI. Open access publication funded by the Max Planck Society. challenges of present-day atomic QED theory. The results presented in this work constitute an important step towards the completion of this project.

A few years ago, the two-loop diagrams featuring two vacuum polarization (VP) loops, as well as those featuring one VP loop and one self-energy (SE) loop, were calculated [25] in the free-fermion loop approximation. The challenging SESE diagrams, which are expected to bring appreciably larger contributions than those of the diagrams considered in the present work, are currently being computed [26,27]. Several VP-VP and VP-SE diagrams were not calculated in Ref. [25], because they vanish in the free VP loop approximation. In this work, we go beyond this approximation and calculate these diagrams, and show that they must be taken into account for heavy ions at the current level of experimental accuracy. We also show that a perturbative calculation of the diagrams examined here is insufficient even for very light ions.

Virtual light-by-light scattering. In a subset of the VP-VP and VP-SE diagrams examined in Ref. [25], the photon from the external magnetic field is attached to a VP loop. This is called the magnetic loop (ML), and vanishes in the free VP loop approximation [20,28]. All diagrams considered in this work contain a ML and another loop, corresponding to either another fermionic pair [electric-loop-magnetic-loop (EL-ML) diagram, see Fig. 1(a); magnetic-loop-after-loop (MLAL) diagram, see Fig. 1(b); and magnetic-loop-next-to-loop (MLNL) diagram, see Fig. 1(c)] or to a virtual photon [self-energymagnetic-loop (SE-ML) diagrams; see Figs. 1(d) and 1(e)]. To the lowest contributing order, the fermion propagator in the ML interacts twice with the Coulomb field of the nucleus [17], so that Delbrück scattering is a subprocess of the overall diagram. The only exception here is the MLNL diagram, for which, to the lowest contributing order, the ML fermion interacts once with the nuclear field and once with the vacuum-polarized nuclear field. This process has never been shown to be well approximated by light-by-light scattering, 
(a)

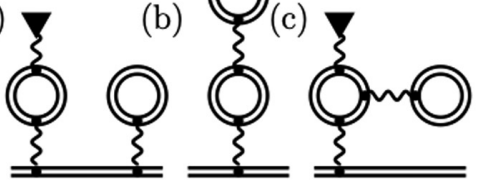

(d)

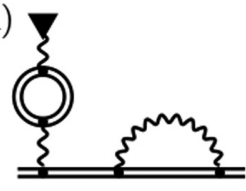

(e)

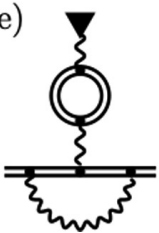

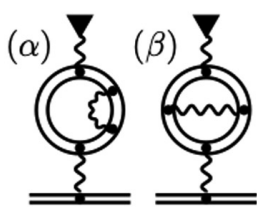

FIG. 1. The diagrams corresponding to the electric-loop-magnetic-loop (a), magnetic-loop-after-loop (b), and magnetic loop-next-toloop (c) contributions, and to the wave-function-type (d) and vertex-type (e) self-energy-magnetic-loop contributions to the $g$ factor of a bound electron. The double line represents the bound electron, internal wavy lines are intermediate photons, while the wavy line terminated by a triangle denotes a photon from the external magnetic field. The SE-in-ML diagrams $(\alpha)$ and $(\beta)$ vanish together with the MLAL diagram (b), in the free-loop approximation, due to the properties of the Källén-Sabry potential. Diagrams (a), (c), (d), and ( $\alpha$ ) each have an equivalent diagram; therefore, their contributions should be counted twice.

and hence the MLNL diagram is not considered in the present work. For high $Z, Z \alpha$ approaches unity, and approximating the contribution of the ML by the (Delbrück) light-by-light scattering process could be insufficient. Nevertheless, this approximation has been found to be satisfactory even for heavy ions [21] in the one-loop case, with a corresponding relative error smaller than $5 \%$ for all $Z \leqslant 92$. This is in agreement with the properties of the Coulomb corrections to Delbrück scattering [21,29]. In what follows, we will make use of the Delbrück-scattered vector potential, which is given [20] in momentum space by

$$
A_{\mathrm{ML} i}(\mathbf{q})=\frac{4 \pi}{\mathbf{q}^{2}} \int \frac{d \mathbf{k}}{(2 \pi)^{3}} M_{j i}(\mathbf{k}, \mathbf{q}) A_{j}(\mathbf{k}),
$$

where we sum over repeated indices. Here $\mathbf{A}$ is the external vector potential, given in momentum space by

$$
\mathbf{A}(\mathbf{k})=\frac{i}{2}(2 \pi)^{3}\left[\mathbf{B} \times \nabla_{\mathbf{k}} \delta(\mathbf{k})\right],
$$

and by $\mathbf{A}(\mathbf{x})=(1 / 2)(\mathbf{B} \times \mathbf{x})$ in configuration space, with $\mathbf{B}$ the homogeneous external magnetic field. Also, $M$ is the (tensor) Delbrück scattering amplitude, which is known in closed form in the partial low-energy limit $|\mathbf{k}| \rightarrow 0$ corresponding to a static, homogeneous magnetic field [20,21], and reads

$$
M_{j i}(\mathbf{k}, \mathbf{q})=\alpha \lambda_{e}^{3}\left[\delta_{j i}(\mathbf{k} \cdot \mathbf{q})-q_{j} k_{i}\right](Z \alpha)^{2} F_{\mathrm{D}}\left(\lambda_{e}|\mathbf{q}|\right),
$$

with the reduced Compton wavelength $\lambda_{e}=\hbar / m_{e} c$. The explicit expression for the Delbrïck scattering function $F_{\mathrm{D}}$ is given in Ref. [21]. It is also helpful to write the expression of the configuration-space Delbrück-scattered vector potential:

$$
\mathbf{A}_{\mathrm{ML}}(\mathbf{x})=\frac{1}{2}(\mathbf{B} \times \mathbf{x}) \Pi_{\mathrm{ML}}\left(\frac{|\mathbf{x}|}{\lambda_{e}}\right),
$$

with the polarization function given by a Bessel transform of the scattering amplitude:

$$
\Pi_{\mathrm{ML}}(u)=4 \frac{\alpha}{\pi}(Z \alpha)^{2} \frac{1}{u^{2}} \int_{0}^{+\infty} d z z u j_{1}(z u) F_{\mathrm{D}}(z) .
$$

The Delbrück-scattered vector potential (4) thus has the same angular structure as the external vector potential.

Calculations. The calculations are carried out in the Furry picture, where the nucleus-electron interaction is included from the outset at all orders, and the wave functions of the electrons are those of the hydrogenlike systems. The contribution from the electric-loop-magnetic-loop diagram of Fig. 1(a) to the $g$ factor of the bound electron can be deduced from the simpler ML diagram studied in Refs. [20,21,28].

We write the EL-ML correction to the $g$ factor as

$$
\begin{aligned}
\Delta g_{a}^{\mathrm{EL}-\mathrm{ML}}= & -\frac{8}{3} \frac{1}{\lambda_{e}} \int_{0}^{+\infty} d r r^{3} \Pi_{\mathrm{ML}}\left(\frac{r}{\lambda_{e}}\right) \\
& \times\left[g_{a}(r) \delta_{\mathrm{VP}} f_{a}(r)+\delta_{\mathrm{VP}} g_{a}(r) f_{a}(r)\right],
\end{aligned}
$$

where $g_{a}$ and $f_{a}$ are the radial parts of the Dirac-Coulomb spinor $\psi_{a}$ [30]. The EL potential is approximated [17] by the Uehling potential and the Uehling-corrected radial wave functions are $\delta_{\mathrm{VP}} g_{a}$ and $\delta_{\mathrm{VP}} f_{a}$. For this diagram, the radial wave functions are computed numerically for finite-size nuclei. To achieve this, the electron is confined in a radial cavity and the dual kinetic balance approach [31] is used to generate numerical spectra and radial wave functions. In our calculations, the nucleus is considered to be a homogeneously charged sphere and the resulting Uehling potential is computed using analytical expressions given in Ref. [32].

The contribution from the magnetic-loop-after-loop diagram of Fig. 1(b) is computed by another modification to the ML diagram. At the free-loop level, the MLAL diagram vanishes, along with the SE-in-ML diagrams [see Figs. 1 $(\alpha)$ and $1(\beta)][25]$. This is due to the low-momentum properties of the (Källén-Sabry) fourth-order VP tensor [33], in the same way that the vanishing of the simpler one-loop ML diagram at the free-loop level $[17,20]$ is due to the low-momentum properties of the (Uehling) second-order VP tensor. Hence, the lowest nonvanishing contribution from the highly challenging SE-in-ML diagrams features a six-photon light-by-light scattering process, and is out of the scope of the present work. The first nonvanishing contribution from the MLAL diagram comes from adding two interactions with the Coulomb field of the nucleus, on the outermost free loop, while keeping the innermost loop free (we call this the scarecrow diagram; see Fig. 2). The diagram wherein the Coulomb photons interact with the innermost loop and the outermost loop is free, has a vanishing contribution. Hence at the lowest nonvanishing order in the VP loops, the contribution of the MLAL diagram 


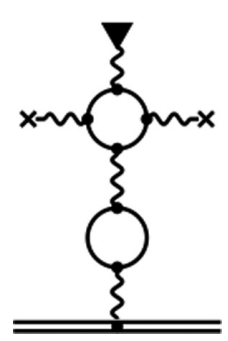

FIG. 2. The "scarecrow" diagram represents the lowest nonvanishing term that contributes to the magnetic loop-after-loop diagram given in Fig. 1(b).

is given by

$$
\Delta g_{a}^{\mathrm{MLAL}}=-\frac{8}{3} \frac{1}{\bar{\lambda}_{e}} \int_{0}^{+\infty} d r r^{3} \Pi_{\mathrm{MLAL}}\left(\frac{r}{\lambda_{e}}\right) f_{a}(r) g_{a}(r),
$$

where the photon interacting with the bound electron has gone through two VP loops and is described by the MLAL polarization function,

$$
\begin{aligned}
\Pi_{\mathrm{MLAL}}(u)= & 4\left(\frac{\alpha}{\pi}\right)^{2}(Z \alpha)^{2} \frac{1}{u^{2}} \\
& \times \int_{0}^{+\infty} d z z u j_{1}(z u) F_{\mathrm{D}}(z) I_{\mathrm{VP}}(z) .
\end{aligned}
$$

Here the one-loop photon VP function is given by [25]

$$
I_{\mathrm{VP}}(z)=z^{2} \int_{0}^{1} d \tau \frac{\tau^{2}\left(1-\frac{\tau^{2}}{3}\right)}{4+\left(1-\tau^{2}\right) z^{2}} .
$$

The MLAL contribution (7) to the $g$ factor is then computed for pointlike nuclei.

The contribution from the wave-function-type self-energymagnetic-loop diagram of Fig. 1(d) can be split into a reducible contribution, wherein the intermediate state in the bound-electron propagator between the SE loop and the ML is the ground state $a=1 s$, and an irreducible contribution, wherein a sum over all intermediate states, excluding the ground state, is performed in that propagator. The irreducible contribution $\Delta g_{a \text { (irr) }}^{\mathrm{SE}-\mathrm{ML}}$ is computed in the same way as the EL-ML diagram: As is the case of the EL, the SE loop does not modify the angular dependence of the wave function of the bound electron, so that, if in Eq. (6), the VP-corrected wave functions are replaced with the SE-corrected wave functions $\delta_{\mathrm{SE}} f_{a}$ and $\delta_{\mathrm{SE}} g_{a}$, we straightforwardly obtain the irreducible correction to the $g$ factor due to the SE-ML diagram. The irreducible contribution was computed with SE-corrected wave functions obtained with the method presented in Ref. [34]. The reducible contribution, on the other hand, is given by

$$
\Delta g_{a(\text { red })}^{\mathrm{SE}-\mathrm{ML}}=\frac{\Delta g_{a}^{\mathrm{ML}}}{g_{a(\mathrm{D})}} \Delta g_{a(\text { red })}^{\mathrm{SE}},
$$

where $g_{a(\mathrm{D})}$ is simply the Breit-Dirac value for the boundelectron $g$ factor [35]. Both the reducible contribution to the one-loop SE correction $\Delta g_{a(\text { red })}^{\mathrm{SE}}$ [19] and the ML correction $\Delta g_{a}^{\mathrm{ML}}[20]$ to the $g$ factor have been investigated previously, therefore, we can directly compute the reducible contribution (10) to the two-loop correction for pointlike nuclei. There is a potential further reducible contribution to the SE-ML diagram, coming from the energy derivative of the electron propagators in the ML, which vanishes because these free propagators do not depend on the energy of the bound electron.

Let us finally turn to the contribution from the vertex SEML diagram of Fig. 1(e). As was the case for the simpler vertex SE one-loop correction [19], we need, for renormalization purposes, to split this diagram into a zero-potential part (whereby the propagator of the electron under the SE loop is taken to be that of the free electron) and a many-potential part (whereby the electron interacts any nonzero number of times with the Coulomb field of the nucleus under the SE loop). The zero-potential term can be treated analytically to a large extent. After renormalization, its general expression is given by

$$
\begin{aligned}
\Delta g_{a(\mathrm{ver})}^{\mathrm{SE}-\mathrm{ML}(0)}= & \frac{2}{\mu_{a} \bar{\lambda}_{e} B} \int \frac{d \mathbf{p}}{(2 \pi)^{3}} \int \frac{d \mathbf{p}^{\prime}}{(2 \pi)^{3}} \bar{\psi}_{a}(\mathbf{p}) \\
& \times \boldsymbol{\Gamma}_{R}\left(p, p^{\prime}\right) \cdot \mathbf{A}_{\mathrm{ML}}\left(\mathbf{p}-\mathbf{p}^{\prime}\right) \psi_{a}\left(\mathbf{p}^{\prime}\right),
\end{aligned}
$$

with $\mu_{a}$ the magnetic projection quantum number, and the Delbrück-scattered vector potential $\mathbf{A}_{\mathrm{ML}}$ given by Eq. (1). The four vectors $p$ and $p^{\prime}$ share the same time component fixed by the energy $\epsilon_{a}$ of the reference state: $p=\left(\epsilon_{a} / c, \mathbf{p}\right)$, $p^{\prime}=\left(\epsilon_{a} / c, \mathbf{p}^{\prime}\right)$, while $\boldsymbol{\Gamma}_{R}$ is the UV-finite part of the free vertex function, studied in detail in Ref. [30]. Performing three of the four angular integrals in Eq. (11) analytically, the zero-potential term in the vertex diagram can be cast as a quadruple integral (a double radial integral, one remaining angular integral, and an integral over a Feynman parameter present from the outset in $\Gamma_{R}$ ), to be performed numerically for pointlike nuclei. The many-potential term, on the other hand, is computed in configuration space, and is treated in a very similar way to the corresponding term in the oneloop SE correction [19,26]: The bound and free electronic Green's function are expanded in partial waves according to the absolute value $|\kappa|$ of the Dirac angular momentum. As can be seen from Eq. (4), the angular structure of the Delbrückscattered vector potential (1) is identical, in configuration space, to that of the external vector potential (2), meaning that the only modification to the calculation concerns the radial integrals. As was the case for the one-loop SE correction, the many-potential term in the vertex diagram features an infrared divergence, that is rigorously canceled by a divergence in the many-potential, reducible contribution [19] [in contrast to the approach of Ref. [19], we do not separate the (finite) one-potential term here]. We computed the sum of the many-potential terms of the reducible and vertex contributions to the SE-ML correction, which is readily finite because of that cancellation. The partial wave summation is truncated at $|\kappa|=20$, with the remaining terms estimated through leastsquares inverse polynomial fitting.

Results. We present numerical results for seven specific hydrogenlike ions, with the nuclear charges $Z=1,2,14,20$, $54,82,92$. The contributions from all diagrams are summarized in Table I.

The EL-ML and irreducible SE-ML corrections were computed for finite-size nuclei, with the nuclear radii [36] taken from Ref. [37]. The MLAL correction, as well as the reducible and vertex SE-ML corrections, were computed for 
TABLE I. Numerical values of the two-loop magnetic-loop correction $\Delta g_{a}^{(2 \mathrm{~L}-\mathrm{ML})}$ to the $g$ factor of hydrogenlike ions $(a=1 s)$ for several nuclear charges $Z$. We give separately the contribution $\Delta g_{a}^{\mathrm{EL}-\mathrm{ML}}$ from the electric-loop-magnetic-loop diagram, the contribution $\Delta g_{a}^{\mathrm{MLAL}}$ from the magnetic-loop-after-loop diagram, and the irreducible $\Delta g_{a(\mathrm{irr})}^{\mathrm{SE}-\mathrm{ML}}$, zero-potential vertex+reducible $\Delta g_{a(\text { ver+red })}^{\mathrm{SE}-\mathrm{ML}(0)}$ and many-potential vertex+reducible $\Delta g_{a \text { (ver+red })}^{\mathrm{SE}-\mathrm{ML}(1+)}$ contributions from the SE-ML diagrams. The total correction is given in the last column. Results are given in units of $10^{-6}$, and powers of 10 are given between square brackets.

\begin{tabular}{|c|c|c|c|c|c|c|c|}
\hline \multirow{2}{*}{$\frac{Z}{1}$} & $\Delta g_{a}^{\mathrm{EL}-\mathrm{ML}}$ & \multicolumn{2}{|c|}{$\Delta g_{a}^{\mathrm{MLAL}}$} & $\Delta g_{a(\mathrm{irr})}^{\mathrm{SE}-\mathrm{ML}}$ & \multirow{2}{*}{$\frac{\Delta g_{a(\mathrm{ver}+\mathrm{red})}^{\mathrm{SE}-\mathrm{ML}(0)}}{-3.0928(1)[-11]}$} & \multirow{2}{*}{$\frac{\Delta g_{a(\text { ver+red })}^{\mathrm{SE}-\mathrm{ML}(1+)}}{-2.600(250)[-11]}$} & \multirow{2}{*}{$\frac{\Delta g_{a}^{(2 \mathrm{~L}-\mathrm{ML})}}{4.92(2.50)[-12]}$} \\
\hline & {$[-14]$} & 3.7 & {$[-14]$} & $-8.6(8)$ & & & \\
\hline 2 & $2.58(1)$ & 2.34 & {$[-12]$} & $-8.9(5) \quad[-12]$ & $-9.4430(8)[-10]$ & $-7.926(6) \quad[-10]$ & $1.477(8) \quad[-10]$ \\
\hline 14 & $3.60(1)$ & 2.42 & {$[-7]$} & $-1.920(8) \quad[-6]$ & $-9.2140(1)[-6]$ & $-8.856(6)$ & $-9.60(10) \quad[-7]$ \\
\hline 20 & $3.189(3) \quad[-6]$ & 1.941 & {$[-6]$} & $-1.662(3) \quad[-5]$ & $-4.2219(1)[-5]$ & $-4.457(3) \quad[-5]$ & $-1.384(4)$ \\
\hline 54 & $1.4344(26)[-3]$ & $6.019(1)$ & {$[-4]$} & $-5.3135(50)[-3]$ & $-1.0331(1)[-3]$ & $-3.3842(24)[-3]$ & $-5.628(6)$ \\
\hline 82 & $2.0982(8)[-2]$ & $6.845(3)$ & {$[-3]$} & $-5.5379(20)[-2]$ & $-4.0125(94)[-3]$ & $-2.0833(22)[-2]$ & $-8.851(3)$ \\
\hline 92 & $4.5676(38)[-2]$ & $1.3648(1$ & 1) $[-2]$ & $-1.0570(5)[-1]$ & $-1.1957(43)[-2]$ & $-3.4565(31)[-2]$ & $-9.290(8)$ \\
\hline
\end{tabular}

pointlike nuclei. The uncertainty of the EL-ML correction is dominated by that on the nuclear radii. The uncertainty of the MLAL correction is given by the estimated finite-nuclear-size correction [38]. The uncertainty of the SE-ML correction is dominated by numerical convergence and, for high $Z(Z=54$, $Z=82$, and $Z=92$; see Table I), by nuclear size corrections.

As anticipated, for instance, in Ref. [25], and as was confirmed in Ref. [24] in the perturbative approach, all corrections obtained here are reliably smaller than the SE-VP [25] and VP-VP [27] corrections from which the ML is absent, especially at low and intermediate $Z$. Nevertheless, for high $Z$, the calculated corrections are large enough to be above the experimental uncertainties of state-of-the-art measurements of the $g$ factor of bound electrons. Indeed the uncertainty reported in the most precise measurements of the $g$ factor $[7,8]$ is of the order of $10^{-11}$. Although these measurements were only performed on lighter ions such as carbon $(Z=6)$ and silicon $(Z=14)$, it is expected that, in the framework of the ALPHATRAP project [2], $g$-factor measurements on heavier ions will be performed at comparable levels of accuracy, meaning that the contributions computed here should be taken into account in an accurate interpretation of these experiments.

At the opposite end of the nuclear charge landscape, we note that our results for the SE-ML contribution approach the prediction of the perturbative approach on Ref. [24] for $Z \rightarrow$ 0 , but that these perturbative results have limited relevance due to large higher-order contributions, which are not captured by the approach of Ref. [24]. This is shown in Fig. 3, where our results for low $Z$ for the SE-ML diagrams [Figs. 1(d) and 1(e)] are parametrized according to

$$
\Delta g^{\mathrm{SE}-\mathrm{ML}}=\left(\frac{\alpha}{\pi}\right)^{2}(Z \alpha)^{5} F_{\mathrm{SE}-\mathrm{ML}}(Z) .
$$

In Ref. [24] the leading term in the function $F_{\mathrm{SE}-\mathrm{ML}}(Z)$ is given by $F_{\mathrm{SE}-\mathrm{ML}}(Z)=(7 / 432) \pi \simeq 0.0509$. However, our numerical results show that a linear term is necessary to capture the behavior of the SE-ML correction to the $g$ factor, even at very low $Z$ :

$$
F_{\mathrm{SE}-\mathrm{ML}}(Z)=a_{5}+a_{6}(Z \alpha),
$$

with the values of the coefficients $a_{5}=0.0505$ (3) and $a_{6}=$ $-0.769(4)$ obtained through a least-squares numerical fit. We thus confirm the result of Ref. [24] through our result for
$F_{\mathrm{SE}-\mathrm{ML}}(Z=0)$. Our numerical results do not suggest any logarithmic terms at the order $(Z \alpha)^{6}$, but we cannot exclude the presence of a logarithm with a numerically small coefficient. We find, however, a large $a_{6}$ coefficient, which induces a $50 \%$ contribution to $F_{\mathrm{SE}-\mathrm{ML}}(Z)$ already at $Z=4$.

Discussion. The two-loop corrections computed here are of the same order of magnitude as the uncertainty in the finite nuclear size correction, as can be checked from Refs. [37,39-41]. As a result, they are arguably mostly relevant within the framework of the "specific" weighted difference between the $g$ factor of $\mathrm{H}$-like and Li-like ions, which allows for the approximate cancellation of nuclear size corrections $[9,10,42]$. For the very heavy Li-like ions of relevance here, these two-loop diagrams can be reasonably well evaluated in the single-electron framework of the present work.

Conclusion. Two-loop QED corrections to the boundelectron $g$ factor involving the magnetic loop were calculated for the first time in the Furry picture. The calculated corrections deviate significantly from the perturbative results [24]

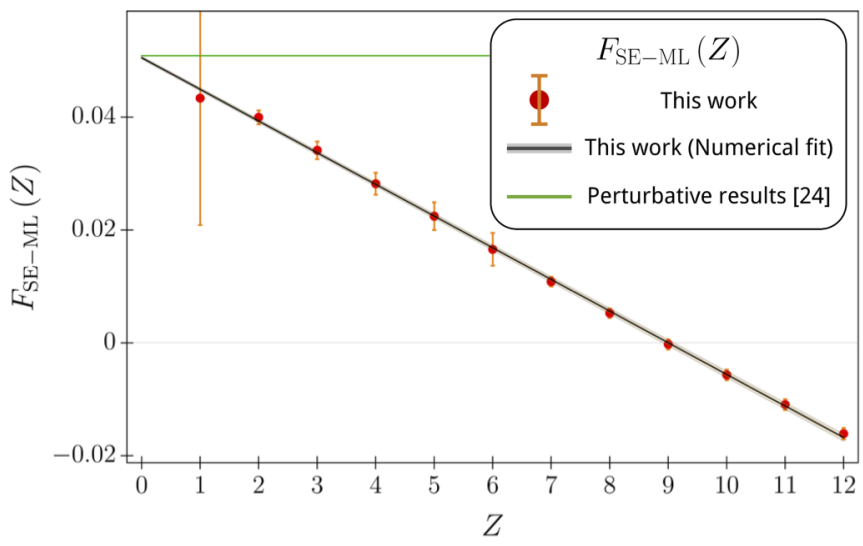

FIG. 3. Numerical values (red dots) of the SE-ML contribution to the $g$ factor of hydrogenlike ions for low nuclear charges, as parametrized through Eq. (12). The error bars (orange) have been artificially magnified by a factor of 4 in order to be visible, except for $Z=1$ where the actual error bar is shown. Our results are fitted (black line) according to Eq. (13), with the shaded gray region around the line corresponding to the uncertainty on the fit parameters. The agreement with the perturbative result (green line) of Ref. [24] is manifest. However, it is seen that the SE-ML contribution markedly departs from the prediction of Ref. [24] even at very low $Z$. 
and are substantially larger than projected experimental uncertainties for heavy hydrogenlike ions, of relevance for tests of QED, searches for new physics, and the determination of fundamental constants.
Acknowledgments. The authors thank Krzysztof Pachucki for helpful discussions. This work is funded by the Deutsche Forschungsgemeinschaft (DFG, German Research Foundation), Project No. 273811115-SFB 1225 (ISOQUANT).
[1] S. Sturm, M. Vogel, F. Köhler-Langes, W. Quint, K. Blaum, and G. Werth, High-precision measurements of the bound electron's magnetic moment, Atoms 5, 4 (2017).

[2] S. Sturm, I. Arapoglou, A. Egl, M. Höcker, S. Kraemer, T. Sailer, B. Tu, A. Weigel, R. Wolf, J. C. López-Urrutia, and K. Blaum, The ALPHATRAP experiment, Eur. Phys. J. Special Topics 227, 1425 (2019).

[3] I. Arapoglou, A. Egl, M. Höcker, T. Sailer, B. Tu, A. Weigel, R. Wolf, H. Cakir, V. A. Yerokhin, N. S. Oreshkina, V. A. Agababaev, A. V. Volotka, D. V. Zinenko, D. A. Glazov, Z. Harman, C. H. Keitel, S. Sturm, and K. Blaum, $g$ Factor of Boronlike Argon ${ }^{40} \mathrm{Ar}^{13+}$, Phys. Rev. Lett. 122, 253001 (2019).

[4] W. Quint, J. Dilling, S. Djekic, H. Häffner, N. Hermanspahn, H.-J. Kluge, G. Marx, R. Moore, D. Rodriguez, J. Schönfelder, G. Sikler, T. Valenzuela, J. Verdú, C. Weber, and G. Werth, HITRAP: A facility for experiments with trapped highly charged ions, Hyp. Int. 132, 457 (2001).

[5] F. Herfurth, Z. Andelkovic, W. Barth, W. Chen, L. A. Dahl, S. Fedotova, P. Gerhard, M. Kaiser, O. K. Kester, H.-J. Kluge, N. Kotovskiy, M. Maier, B. Maßß, D. Neidherr, W. Quint, U. Ratzinger, A. Reiter, A. Schempp, Th. Stöhlker, H. Vormann, G. Vorobjev, and S. Yaramyshev for the HITRAP collaboration, The HITRAP facility for slow highly charged ions, Phys. Scr. 2015, 014065 (2015).

[6] M. Vogel, M. S. Ebrahimi, E. Guo, A. Khodaparast, G. Birk, and W. Quint, Electron magnetic moment in highly charged ions: The ARTEMIS experiment, Ann. Phys. (Berl.) 531, 1800211 (2019).

[7] S. Sturm, A. Wagner, M. Kretzschmar, W. Quint, G. Werth, and K. Blaum, $g$ factor measurement of hydrogenlike ${ }^{28} \mathrm{Si}^{13+}$ as a challenge to QED calculations, Phys. Rev. A 87, 030501(R) (2013).

[8] S. Sturm, F. Köhler, J. Zatorski, A. Wagner, Z. Harman, G. Werth, W. Quint, C. H. Keitel, and K. Blaum, High-precision measurement of the atomic mass of the electron, Nature (London) 506, 467 (2014).

[9] V. M. Shabaev, D. A. Glazov, N. S. Oreshkina, A. V. Volotka, G. Plunien, H. J. Kluge, and W. Quint, $g$-Factor of Heavy Ions: A New Access to the Fine Structure Constant, Phys. Rev. Lett. 96, 253002 (2006).

[10] V. A. Yerokhin, E. Berseneva, Z. Harman, I. I. Tupitsyn, and C. H. Keitel, $g$ Factor of Light Ions for an Improved Determination of the Fine-Structure Constant, Phys. Rev. Lett. 116, 100801 (2016).

[11] H. Cakir, N. S. Oreshkina, I. A. Valuev, V. Debierre, V. A. Yerokhin, C. H. Keitel, and Z. Harman, Improved access to the fine-structure constant with the simplest atomic systems, arXiv:2006.14261.

[12] S. Sturm, A. Wagner, B. Schabinger, J. Zatorski, Z. Harman, W. Quint, G. Werth, C. H. Keitel, and K. Blaum, $g$ Factor of Hydrogenlike ${ }^{28} \mathrm{Si}^{13+}$, Phys. Rev. Lett. 107, 023002 (2011).
[13] V. M. Shabaev, D. A. Glazov, G. Plunien, and A. V. Volotka, Theory of bound-electron $g$ factor in highly charged ions, J. Phys. Chem. Ref. Data 44, 031205 (2015).

[14] Z. Harman, B. Sikora, V. A. Yerokhin, H. Cakir, V. Debierre, N. Michel, N. S. Oreshkina, N. A. Belov, J. Zatorski, and C. H. Keitel, The $g$ factor of highly charged ions, J. Phys.: Conf. Ser. 1138, 012002 (2018).

[15] P. Indelicato, QED tests with highly charged ions, J. Phys. B 52, 232001 (2015)

[16] V. Debierre, C. H. Keitel, and Z. Harman, Fifth-force search with the bound-electron $g$ factor, Phys. Lett. B 807, 135527 (2020).

[17] T. Beier, I. Lindgren, H. Persson, S. Salomonson, P. Sunnergren, H. Häffner, and N. Hermanspahn, $g_{j}$ factor of an electron bound in a hydrogenlike ion, Phys. Rev. A 62, 032510 (2000).

[18] S. G. Karshenboim, V. G. Ivanov, and V. M. Shabaev, Vacuum polarization in a hydrogen-like relativistic atom: $g$ factor of a bound electron, J. Exp. Theor. Phys. 93, 477 (2001).

[19] V. A. Yerokhin, P. Indelicato, and V. M. Shabaev, Evaluation of the self-energy correction to the $g$ factor of $s$ states in H-like ions, Phys. Rev. A 69, 052503 (2004).

[20] R. N. Lee, A. I. Milstein, I. S. Terekhov, and S. G. Karshenboim, Virtual light-by-light scattering and the $g$ factor of a bound electron, Phys. Rev. A 71, 052501 (2005).

[21] R. N. Lee, A. I. Milstein, I. S. Terekhov, and S. G. Karshenboim, $g$ factor of the bound electron and muon, Can. J. Phys. 85, 541 (2007).

[22] K. Pachucki, A. Czarnecki, U. D. Jentschura, and V. A. Yerokhin, Complete two-loop correction to the bound-electron $g$ factor, Phys. Rev. A 72, 022108 (2005).

[23] A. Czarnecki and R. Szafron, Light-by-light scattering in the Lamb shift and the bound electron $g$ factor, Phys. Rev. A 94, 060501(R) (2016).

[24] A. Czarnecki, M. Dowling, J. Piclum, and R. Szafron, TwoLoop Binding Corrections to the Electron Gyromagnetic Factor, Phys. Rev. Lett. 120, 043203 (2018).

[25] V. A. Yerokhin and Z. Harman, Two-loop QED corrections with closed fermion loops for the bound-electron $g$ factor, Phys. Rev. A 88, 042502 (2013).

[26] B. Sikora, Quantum field theory of the $g$-factor of bound systems, Ph.D thesis, Heidelberg Universität, 2018.

[27] B. Sikora, V. A. Yerokhin, N. S. Oreshkina, H. Cakir, C. H. Keitel, and Z. Harman, Theory of the two-loop self-energy correction to the $g$-factor in non-perturbative Coulomb fields, Phys. Rev. Research 2, 012002(R) (2020).

[28] S. G. Karshenboim and A. I. Milstein, Delbrück scattering and the $g$-factor of a bound electron, Phys. Lett. B 549, 321 (2002).

[29] A. I. Milstein and M. Schumacher, Present status of Delbrück scattering, Phys. Rep. 243, 183 (1994). 
[30] V. A. Yerokhin and V. M. Shabaev, First-order self-energy correction in hydrogenlike systems, Phys. Rev. A 60, 800 (1999).

[31] V. M. Shabaev, I. I. Tupitsyn, V. A. Yerokhin, G. Plunien, and G. Soff, Dual Kinetic Balance Approach to Basis-Set Expansions for the Dirac Equation, Phys. Rev. Lett. 93, 130405 (2004).

[32] S. Klarsfeld, Analytical expressions for the evaluation of vacuum-polarization potentials in muonic atoms, Phys. Lett. B 66, 86 (1977).

[33] G. Källén and A. Sabry, Fourth order vacuum polarization, Mat. Fys. Medd. Dan. Vid. Selesk 29, 1 (1955).

[34] N. S. Oreshkina, H. Cakir, B. Sikora, V. A. Yerokhin, V. Debierre, Z. Harman, and C. H. Keitel, Self-energy-corrected Dirac wave functions for advanced QED calculations in highly charged ions, Phys. Rev. A 101, 032511 (2020).

[35] G. Breit, The magnetic moment of the electron, Nature (London) 122, 649 (1928).

[36] Since the nuclear radii also depend on the isotope considered, we clarify here that we use the most abundant isotopes ${ }_{1}^{1} \mathrm{H}$, ${ }_{2}^{4} \mathrm{He}^{+},{ }_{14}^{28} \mathrm{Si}^{13+},{ }_{20}^{40} \mathrm{Ca}^{19+},{ }_{54}^{132} \mathrm{Xe}^{53+},{ }_{82}^{208} \mathrm{~Pb}^{81+},{ }_{92}^{238} \mathrm{U}^{91+}$. In the case of xenon, the isotope used here has the radius which is known with the best accuracy [37].
[37] I. Angeli and K. P. Marinova, Table of experimental nuclear ground state charge radii: An update, At. Data Nucl. Data Tables 99, 69 (2013).

[38] The corresponding nuclear size uncertainties are evaluated by multiplying the calculated corrections for pointlike nuclei, with the leading finite nuclear size correction to the $g$ factor $[39,43]$, and dividing by the Breit-Dirac value for the $g$ factor.

[39] V. M. Shabaev, Finite nuclear size corrections to the energy levels of the multicharged ions, J. Phys. B: At. Mol. Opt. Phys. 26, 1103 (1993).

[40] J. Zatorski, N. S. Oreshkina, C. H. Keitel, and Z. Harman, Nuclear Shape Effect on the $g$ Factor of Hydrogenlike Ions, Phys. Rev. Lett. 108, 063005 (2012).

[41] N. Michel, J. Zatorski, N. S. Oreshkina, and C. H. Keitel, Nonperturbative analysis of nuclear shape effects on the bound electron $g$ factor, Phys. Rev. A 99, 012505 (2019).

[42] V. A. Yerokhin, E. Berseneva, Z. Harman, I. I. Tupitsyn, and C. H. Keitel, Weighted difference of $g$ factors of light Li-like and $\mathrm{H}$-like ions for an improved determination of the finestructure constant, Phys. Rev. A 94, 022502 (2016).

[43] D. A. Glazov and V. M. Shabaev, Finite nuclear size correction to the bound-electron $g$ factor in a hydrogenlike atom, Phys. Lett. A 297, 408 (2002). 